\title{
Instructional approaches to foster third graders' adaptive use of strategies: an experimental study on the effects of two learning environments on multi-digit addition and subtraction
}

\author{
Aiso Heinze $^{1}\left(\right.$ D $\cdot$ Julia Arend $^{2} \cdot$ Meike Gruessing $^{3} \cdot$ Frank Lipowsky $^{2}$
}

Received: 9 October 2017 / Accepted: 18 May 2018 / Published online: 24 May 2018

(C) The Author(s) 2018

\begin{abstract}
The adaptive use of strategies, that is selecting a strategy which allows an efficient solution for a given problem, can be considered as an important individual ability relevant in various domains. Based on models of subjects' skills of adaptive use of strategies, two idealized instructional approaches are suggested to foster students in their strategy development. The explicit approach aims at reducing cognitive load by demonstrating and practicing strategies combined with an explicit identification of criteria for strategy efficiency by contrasting problem solutions. The implicit approach capitalizes on the generation effect and stimulates students to generate their own strategies and efficiency criteria based on the analysis of task characteristics and the comparison of problem solutions. In a 1-week experimental study (16 lessons) with 73 third-graders, we examined the effectiveness of these instructional approaches in the domain of multi-digit addition and subtraction. Results from post- and two follow-up tests after 3 and 8 months did not yield different effects of the two approaches on students' skills in adaptive use of strategies. A comparison of strategies used by the students showed that the students of the explicit approach more frequently applied complex strategies whereas the students from the implicit approach showed a more sustainable use of self-generated strategies. Hence, for the adaptive use of those strategies students are able to generate, the implicit approach turned out to be more effective than the explicit approach. However, this generation effect does not hold for strategies which are too complex to be generated by students.
\end{abstract}

Keywords Adaptive use of strategies $\cdot$ Instructional approaches $\cdot$ Multi-digit addition and subtraction

Aiso Heinze

heinze@ipn.uni-kiel.de

1 Department of Mathematics Education, Leibniz Institute for Science and Mathematics Education (IPN), Olshausenstrasse 62, $24118 \mathrm{Kiel}$, Germany

2 Department of Educational Science, University of Kassel, Nora Platiel Straße 1, 34109 Kassel, Germany

3 Faculty of Science and Social Science, University of Vechta, Kreuzweg 3-5, 49377 Vechta, Germany 


\section{Introduction}

Cognitive variability - specifically the ability to adaptively apply a strategy to characteristics of a given problem in a given situation so that an efficient solution is possible-can be considered as an important part of human cognition. Accordingly, the understanding of how subjects acquire cognitive variability is an important goal of research on individual learning (cf. Lemaire and Siegler 1995) and, hence, of particular interest for instructional research. In the last decades, empirical research showed that already primary school children are able to solve problems by adaptively using strategies depending on the characteristics of tasks or the self-perception of their strategy skills (e.g., Klayman 1985; Gardner and Rogoff 1990; Crowley and Siegler 1993). Simultaneously, there is empirical evidence that children do not necessarily show an adaptive use of strategies concerning task characteristics, even when they know relevant efficient strategies (e.g., Siegler 1996). This phenomenon is well-known for arithmetic where many children use one favorite strategy to solve all addition or all subtraction problems (e.g., Heinze et al. 2009). This restriction to one favorite strategy might specifically occur in domains in which universal and task-specific strategies exist. As elaborated below, universal strategies can be applied to all problems of a domain (i.e., all subtraction problems) with a similar efficiency whereas task-specific strategies are highly efficient for a specific class of problems and hardly efficient for other problems.

From the perspective of instructional science, an important question is how the individual skill of adaptive use of strategies can be fostered by instruction. Some instructional approaches - especially in mathematics education - frequently focus on teaching activities asking students to invent their own strategies instead of presenting strategies to students (e.g., Threlfall 2009). The idea that students can find efficient strategies on their own can be derived from assumptions on children's strategy development (e.g., Siegler 2003). However, there is still a lack of empirical evidence for the effectiveness of learning environments in this field of research. Though we know that already children can invent strategies on their own, it is an open question whether they are also able to invent complex taskspecific strategies. Moreover, for demanding requirements like the adaptive use of strategies it is unclear whether the invention of strategies by students leads to more sustainable skills - in the sense of the generation effect-than an explicit teaching of strategies based on a teacher presentation. To address these questions, we conducted an experimental study with primary school children and compared two instructional approaches. We chose the domain of arithmetic (addition and subtraction with three-digit numbers) which provides a sound basis of research results on children's strategy skills for our study.

\section{Theoretical background and empirical findings}

\section{Basic definitions: adaptive use of strategies and accurate use of strategies}

There is no consistent use of the concepts strategy efficiency, strategy flexibility, and strategy adaptivity in the research literature. Depending on the underlying models, sometimes strategy flexibility and strategy adaptivity are used synonymously and different frames of reference exist for strategy efficiency (Verschaffel et al. 2009). Many research studies follow the definitions as presented in the model of strategy change by Lemaire and Siegler 
(1995) which strongly relies on an individual frame of reference. Lemaire and Siegler (1995) define strategy efficiency by the accuracy and speed of an individual's strategy execution. Accordingly, strategy flexibility of an individual goes back to individual strategy efficiency, that is, for a given task the individual applies the strategy from its strategy repertoire which allows her/him the fastest accurate solution. In our research, we build on this idea but combine the individual frame of reference with task-specific criteria for strategy efficiency. We want to examine a teaching experiment to improve students' skills in strategy use. Here, the (ideal) teaching goal is that a student uses a strategy to solve a given task that satisfies task-specific criteria as well as individual criteria for strategy efficiency. We distinguish between two aspects concerning students' strategy use: the taskspecific efficiency of students' solutions, denoted by adaptive use of strategies, and the accuracy of these solutions, denoted by accurate use of strategies. Hence, adaptive use of a strategy by an individual means that, for a given task a strategy is used (accurately or inaccurately) which, from a normative perspective, is an efficient solution to this task (or in case of inaccurate strategy processing: would be an efficient solution when correcting slips occurred in intermediate calculation steps). By taking into account the normative task-specific approach for strategy efficiency we follow the approach of several other studies (e.g., Beishuizen 1993; Blöte et al. 2000, 2001; Heinze et al. 2009; Klein et al. 1998; Torbeyns et al. 2009a). The main reason to distinguish between adaptive and accurate use of strategies in our experimental study is the fact that students should learn new strategies and their adaptive and accurate uses. In this case it is also a learning progress if a student chooses an efficient strategy for a given problem based on task-specific criteria (i.e., adaptive use of a strategy) and gets a wrong result due to a slip during strategy processing (i.e., inaccurate use of a strategy). Obviously, the final teaching goal covers both the adaptive and accurate use of strategies.

\section{Theoretical perspectives on the teaching and learning of adaptive use of strategies}

In the last decades several researchers elaborated on the question how to model students' skills in the adaptive use of strategies and its development (e.g., models in Symons et al. 1989; Baroody 2003). Based on the strategy choice model (Lemaire and Siegler 1995; Siegler 1996), an individual's adaptive use of strategy can be described by selecting an appropriate strategy from the individual's strategy repertoire. The individual's decision on the appropriateness depends on how frequently the individual applied the strategies of the strategy repertoire for certain problems (strategy distribution, which strengthen the association between problem type and strategy), and the individual accuracy and speed of the strategy execution. Siegler (1996) describes the development of students' skills in adaptive use of strategy using his model of overlapping waves. This model is based on the assumptions that students use strategies of different efficiency over a certain period of time and students' experience in strategy use leads to the discovery of advanced strategies. Based on empirical evidence of a series of studies and computer-based models like the Strategy Choice and Discovery Simulation Model (Shrager and Siegler 1998), Chen and Siegler (2000) postulate five components for the discovery of new strategies: strategy acquisition, mapping the strategy onto novel problems, strengthening the strategy for given types of problems, refining strategy choice based on the existing strategy repertoire, and increasing effectiveness of strategy execution. The results of Siegler and colleagues suggest that strategy discovery takes place on the basis of suitable tasks. These allow students to experience 
strategy execution which increases strategy fluency and reduces cognitive load. The available cognitive resources give the opportunity to perform metacognitive processes like examining strategy efficiency which might result in discovering new strategies or strengthening the association between existing strategies and problem types. According to this model of strategy discovery, it is not necessary to teach strategies or adaptive use of strategies explicitly (i.e., presenting strategies and efficiency criteria to the students) because new strategies are invented when children are exposed to with suitable problems (Siegler 2003). According to Siegler (2003) it is not even necessary to provide specific situations for discovery learning (see also De Smedt et al. 2010; Torbeyns et al. 2009a).

Alternative models about students' adaptive use of strategies provide different descriptions and explanations but they also do not recommend (they may even reject) an explicit presentation of strategies and their adaptive use. In the domain of arithmetic, for example, the schema-based view of Baroody and Ginsburg (1986) stresses the important role of conceptual knowledge on numbers (see also Baroody and Tiilikainen 2003). Concerning the strategy discovery, Baroody and colleagues follow the idea of an iterative development of conceptual and procedural knowledge so that both will be intertwined (Baroody and Ginsburg 1986). The recommended teaching approach (denoted by "investigative approach") is described as ,,a teacher mentors children, guiding their meaningful construction of procedures and concepts and the development of mathematical thinking" by indirect means (Baroody 2003, p. 22).

A different perspective is taken by Threlfall $(2002,2009)$. He argues that the strategychoice model by Siegler and colleagues is too restricted for an explanatory model describing the diversity of strategies children invent and use. In particular, Threlfall criticizes the idea of a strategy repertoire from which a strategy is chosen for a given problem. Instead he describes children's approaches to new problems as strategy emergence: children do not follow a fixed schema but analyze the relation of concepts in a given problem to decide the first step for a possible solution and then proceed step by step based on the intermediate results. This might result in (exploratory) partial solutions which, finally, constitute a solution to the problem. Hence, the solution is not based on a holistically selected strategy from a strategy repertoire. Instead it emerges from a specific interaction between problem characteristics and individual conceptual knowledge (Threlfall 2009). Following this strategy-emergence model, an explicit teaching of strategies and its adaptive use is also not appropriate for the learning of adaptive use of strategies. It should be avoided because it is not creative and does not involve the analysis of problem characteristics (Threlfall 2009). Instead it seems to be more effective that students frequently experience processes of solving computation problems by developing solutions and comparing their efficiency (Threlfall 2002). According to Gravemeijer (2004) such a repeated analysis of problem characteristics in relation to the efficiency of the solutions allows the students to generalize from a situation-specific consideration of a specific problem and to associate strategies with problem types.

Though the previously described models differ in their assumption how students process a problem (strategy choice vs. strategy emergence), they coincide in their conclusion concerning instruction. In both cases it is suggested that students generate strategies on their own and learn the adaptive use of strategies without demonstration through the teacher or material. The possibility that generation of the learning content by students is an effective instructional approach, which can be more effective than learning by presentation of the content, is described as "generation effect" (Slamecka and Graf 1978). The generation effect was frequently replicated in laboratory settings with medium and large effect sizes for various content areas (Bertsch et al. 2007). A widely accepted explanation of this effect 
Table 1 Main types of computation strategies with examples

\begin{tabular}{lllll}
\hline Jump strategies & Split strategies & Compensation strategies & Simplifying strategies & $\begin{array}{l}\text { Indirect } \\
\text { subtraction } \\
\text { strategies }\end{array}$ \\
\hline $123+456=579$ & $123+456=579$ & $527+398=925$ & $527+398=925$ & $701-698=3$ \\
$123+400=523$ & $100+400=500$ & $527+400=927$ & $525+400=925$ & $698+3=701$ \\
$523+50=573$ & $20+50=70$ & $927-2=925$ & & \\
$573+6=579$ & $3+6=9$ & & & \\
& $500+70+9=579$ & & & \\
& & & & \\
&
\end{tabular}

The indirect subtraction strategies, e.g., indirect addition, are for subtraction problems only

The italic numbers represent the results of the problems

is that the cognitive processes used to generate a learning content in the learning process match the cognitive processes when subsequently applying this content in further situations (e.g., McNamara and Healy 2000). In case of learners with low prior knowledge, it turned out that not only facts could be remembered better when generated by a subject but also subjects' ability to transfer memorized procedures to unstudied problems of the same type increased (e.g., Rittle-Johnson and Kmicikewycz 2008).

A necessary condition for the generation effect is that students are able to generate the target information correctly. Hence, it can be questioned whether the generation of a complex learning content (like adaptive use of strategies) is an effective instructional approach because students might not be able to generate the information at all or they generate and memorize incorrect facts or procedures. To date, most of the studies supporting the generation effect considered simple learning contents, that is simple facts or procedures. Chen et al. $(2015,2016)$ compared learning by generation with learning by worked examples varying simple and complex contents. Their studies showed that for complex contents learning by worked examples (i.e., by demonstration) is more effective than learning by generation whereas for simple contents the reverse holds. For students with high prior knowledge the expertise-reversal effect occurred, that is these students benefit more from learning by generation in the case of complex contents than from learning by worked examples.

If we consider the learning of adaptive use of strategies for primary school children, then the learning content can be classified as complex. As previously described in the model of Siegler and colleagues, the students have to learn a combination of different aspects like different strategies, their accurate and fluent use, their flexible use as well as criteria for which tasks a strategy is efficient. The findings of Rittle-Johnson and Kmicikewycz (2008) suggest a generation effect for the acquisition of strategies and their accurate and fluent use, whereas the findings of Chen et al. $(2015,2016)$ indicate that a teacher demonstration might be more effective in the complex case of the adaptive use of strategies. Hence, it is an open question whether instruction is more effective when students invent strategies and learn the adaptive use of strategies (as suggested) than a demonstration through explicit teaching. 


\section{Empirical results on the teaching and learning of adaptive use of addition and subtraction strategies}

In this section we will present empirical findings on primary school children's skills in adaptive use of strategies specifically for multi-digit addition and subtraction problemsthe domain we addressed in our own study. The advantage of the restriction to a specific domain is that there exist categorizations of the relevant strategies from previous studies (e.g., Threlfall 2002, pp. 33ff.). For our study presented below, we use the categorization described in Table 1. It distinguishes five main types of strategies for addition and subtraction problems, each type covers several strategies. For example, the jump strategy type encompass the two strategies which successively add (i) the hundreds, tens and units or (ii) the units, tens and hundreds of the second summand to the first summand, the two strategies (iii, iv) which analogously decompose only the first summand and successively add these components to the second summand, and finally, in case of subtraction, the two strategies which successively subtract (v) the hundreds, tens and units or (vi) the units, tens and hundreds of the subtrahend from the minuend. All strategies represented by the strategy types in Table 1 are ideal-typical strategies in the sense that children obviously are quite creative and invent further strategies, especially by combining two strategies of different types (e.g., Selter 2001).

As elaborated in the previous section, the different models of adaptive use of strategies do not imply the necessity of an explicit teaching of strategies or their adaptive use. Instead it is assumed that continuous experience in solving problems combined with the acquisition of conceptual knowledge will result in the generation of new strategies and its adaptive application. Empirical results on adaptive use of strategies of primary school children from different countries challenge this assumption (e.g., Carpenter et al. 1997; Csíkos 2016; Selter 2001; Torbeyns et al. 2006; Heinze et al. 2009; Torbeyns et al. 2009a, b). It turns out that most children do not use addition and subtraction strategies adaptively with respect to task characteristics (e.g., Heinze et al. 2009; Torbeyns et al. 2009a, b; Torbeyns and Verschaffel 2016). Instead many students have a favourite strategy which they use as a standard procedure (German children frequently prefer the jump strategy for subtraction tasks and the split strategy for addition tasks, Heinze et al. 2009, and they almost solely use the standard algorithms after their introduction, Selter 2001). Children rarely apply task-specific strategies like indirect addition (Csíkos 2016; Heinze et al. 2009; Selter 2001; Torbeyns et al. 2009a) so that it can be called into question whether such strategies can be self-generated in grade 2 or 3.

Based on studies following the choice/no choice paradigm, Torbeyns and colleagues showed that students do not use advantageous strategies for specific tasks in the choice condition though they know and were able to apply these strategies in the no-choice condition (e.g., Torbeyns et al. 2009b; Torbeyns and Verschaffel 2016). Referring to the influence of the social and cultural context (Verschaffel et al. 2009), it is assumed that children's participation in specific socio-mathematical practices encourages children to develop only specific strategies and to apply mainly these highly valued strategies (Torbeyns et al. 2009a). This implies in particular that the discovery of new strategies and the adaptive use of strategies are not only based on individual characteristics of the children but also on the social context of the learning environment. Even when a negative influence of the social context can be excluded, it can be called into question whether children invent highly efficient strategies for given problems on their own (see for example the study of De Smedt et al. (2010) presented below). 
To date there are only a few experimental studies which examined the influence of instructional conditions on children's adaptive use of strategies in arithmetic. In a 1-year longitudinal study with ten second-grade classes Klein et al. (1998) compared two teaching approaches (Realistic Program Design, RPD vs. Gradual Program Design, GDP). These teaching approaches differ in their extent of explicitly addressing computation strategies and their adaptive use (see also Blöte et al. 2001). The GDP focused on teaching the jump strategy (cf. Table 1) for almost the entire school year before at the end of that period introducing the split strategy (cf. Table 1) and comparing strategies regarding their efficiency. In the RPD children were asked to invent their own strategies and they were always encouraged to use and compare different strategies. If they were not able to develop specific strategies the teacher explicitly introduced these strategies and children got time to practice these new strategies. The results of the comparison indicate that children in the RPD group outperformed the children of the GDP group concerning the adaptive use of strategies, whereas no significant difference occurred for the accuracy of strategy application (Klein et al. 1998).

Klein, Blöte and colleagues concluded that it is more effective to teach students an adaptive use of strategies from the beginning (i.e., compare several strategies shortly after their introduction) instead of teaching and practicing only one strategy for some time before supplementing it with the next strategy (Blöte et al. 2001). This conclusion is supported by studies of Rittle-Johnson and colleagues with seventh and eighth graders on learning of equation solving in algebra (Durkin et al. 2017; Rittle-Johnson et al. 2012; Rittle-Johnson and Star 2007). They compared different conditions of the explicit teaching of strategies by worked examples. It turned out that students who immediately compared different solution strategies for a given task made greater gains in an adaptive use of strategies than students who learned and reflected the strategies sequentially without direct comparison.

The previously mentioned study of De Smedt et al. (2010), which focuses the indirect addition strategy, compared two learning environments for third-graders. The indirect addition strategy was demonstrated by the teacher in the explicit learning environment whereas the children in the implicit learning environment processed a large number of subtractions suggesting indirect addition as a useful and quick computation strategy. From the beginning a comparison of strategies was encouraged in both learning environments. It turned out that in the intermediate tests during the intervention none of the children in the implicit learning environment used the indirect addition strategy and even in a transfer session this strategy was hardly applied. Hence, it seemed that children were not able to invent this strategy since it is too challenging. Children from the explicit learning environment used the indirect addition strategy more often though the frequency was still quite low.

\section{The present study}

The results presented in the previous sections give no clear indication about the theoretically assumed effectiveness of the implicit instruction of an adaptive use of strategies. To our knowledge, only the study of De Smedt et al. (2010) directly compared an implicit and explicit instructional approach. The results of this study indicated a tendency for the explicit approach but the findings can hardly be generalized. Due to the aim of this study it was restricted to the specific and challenging strategy of indirect addition. In the study of Klein et al. (1998) the RPD condition covered features from an implicit teaching approach (in contrast to the GDP condition). However, in the RPD condition children were encouraged to compare strategies immediately whereas in the 
GPD condition the strategies were introduced consecutively and one particular strategy was emphasized. Hence, in addition to the implicit/explicit teaching approach there was a confounding second condition which could have influenced the differences between RPD versus GDP. Finally, a cross-sectional study of Heinze et al. (2009) used textbook information as an indicator for the features of the learning environments. The results indicate that German third graders taught by a textbook series following an implicit teaching approach showed a significantly better adaptive use of strategies than students taught by a textbook series that followed an explicit approach. Both textbook series addressed the comparison of different strategies. However, no data about the actual classroom teaching was included in this study so that it is not clear whether the teachers followed the intention of the textbooks.

Summarizing existing empirical results, it remains unclear whether a learning environment addressing strategies and adaptive use of strategies implicitly (in the sense of learning by generation) is more effective than an explicit teaching of strategies and their adaptive use (in the sense of learning by presentation). This particularly encompasses the question on long-term effects which can be expected when the generation effect holds for the learning of strategies and their adaptive use. Moreover, more research is required regarding the question whether children are able to invent demanding strategies like for example indirect addition or simplifying (cf. Table 1) on their own.

The present study compared the effects of learning environments addressing strategies and their adaptive use implicitly and explicitly respectively. In an experimental study we implemented two environments for learning the adaptive use of strategies for addition and subtraction problems for numbers up to 1000. Both environments offered the same type of computation problems suggesting specific strategies for an efficient solution and asked the children to compare strategies from the beginning. In the explicit environment the teacher presented specific strategies (cf. Table 1) and made sure that all children practiced these strategies. In the implicit environment the children analyzed number characteristics of given addition and subtraction problems, generated their own strategies and discussed the efficiency of their self-invented strategies (moderated by the teacher). The teacher did not introduce new strategies and did not explicitly show a preference for one strategy. For both learning environments we investigated short- as well as long-term effects (after 3 and 8 months). Taking into account the results of Selter (2001) and of Torbeyns and Verschaffel (2016) we specifically analyzed the sustainability of children's adaptive use of strategies after the introduction of the dominant written algorithms in the regular mathematics instruction.

Based on these conditions we tested several hypotheses. Following the theoretical assumptions (Sect. "Theoretical perspectives on the teaching and learning of adaptive use of strategies") we predicted - as a short-term and as a long-term effect-that children in the implicit learning environment would show an overall better adaptive use of strategies than the children in the explicit learning environment (Hypothesis 1). Similar to the results of De Smedt et al. (2010) and Klein et al. (1998) we did not expect differences between the effects of the two learning environments concerning the accuracy (Hypothesis 2). Concerning the specific types of strategies the children use, we expected that children taught in the explicit learning environment would more frequently use the task-specific strategies of the types indirect addition and simplifying (cf. Table 1) than children from the implicit learning environment (Hypothesis 3). It is a very demanding task for third graders to self-invent these strategies-even when suitable tasks are provided (cf. De Smedt et al. (2010) for the special case of the indirect addition strategy). Finally, we predicted that the group of children taught by the implicit learning 
environment would be more sustainable in the use of task-specific strategy types than children taught by the explicit approach (Hypothesis 4). This hypothesis is rooted in the assumptions that the children in the implicit group would have self-invented the strategies by repeated analysis of problem characteristics based on their numerical knowledge which supports an association of strategies with problem types (cf. Threlfall 2002; Gravemeijer 2004) so that a generation effect occurs (McNamara and Healy 2000).

\section{Method}

\section{Design}

The study followed a controlled randomized experimental design with two experimental groups representing the implicit and the explicit learning environment on adaptive use of strategies. The intervention was organized as a 1-week course with third-graders at our research institute during the two-week fall break. The overall intervention time was equivalent to 16 schools lessons (45 min each) distributed over 5 days. The break time between the lessons was reserved for playing (non-mathematical) games and for lunch. The lessons were taught by two trained research assistants following ideal-typical teaching scripts of the explicit and the implicit approach (see Sect. "Treatments"). We had two student groups for each condition (one group was taught in the first and one in the second holiday week) to limit the group size. To control for teacher effects, both teachers taught each approach once. Data for adaptive and accurate use of strategies was collected by trained university assistants with a pre-test 2 weeks before the intervention (T1), a post-test (T2) at the end of the intervention and two follow-up tests after 3 months (T3) and after 8 months (T4) at the end of the school year. Between T3 and T4 the students learned the standard algorithms for addition and subtraction in their regular mathematics classrooms. In addition, we collected data for general cognitive abilities at $\mathrm{T} 1$ as a covariate.

\section{Participants}

The sample of the study comprised 73 third-graders (9-10 years old) from 17 classes of German primary schools. For sample recruitment we distributed an invitation for a 5-days holiday course in mathematics to Grade 3 classes of 8 schools. Parents got the information that the course is part of a research study, is free of charge (including food) and that daily participation is obligatory from 9:00 a.m. to 4:00 p.m. Moreover, they had to consent to the data collection based on information about the intervention and its investigation. Altogether, 79 third-graders (9-10 years old) were registered by their parents for the holiday course. Six children were excluded from the analysis for this study because their pretest results at T1 showed that they either already reached the intervention goal (i.e., they already solved almost all test items highly adaptively) or they only used written algorithms which students are expected to learn in the second semester of Grade 3 and which hinder children from using strategies adaptively (Selter 2001; Torbeyns and Verschaffel 2016). For the experiment the children were randomly allocated to one of the two conditions. A few children were reallocated at the beginning of the intervention due to specific requests of some children which wanted to be 
in the same group as their friends. Finally, 38 children participated in the explicit condition and 35 children in the implicit condition.

We were able to administer the tests for adaptive and accurate use of strategies at T1 and T3 not only to the intervention groups but to all children in the 17 classes (at T1 only in 15 classes). Hence, we have complete data at T1 and T3 of 179 classmates of the children from our intervention group which we used to examine the sample selection and to conduct an intervention check (see Sect. "Results"; there is no data at T2 for the classmates since T2 was during fall break).

\section{Procedure and materials}

\section{Treatments}

The intervention was organized as a 5-days course at our research institute. The plan to conduct the intervention outside of a school context and to collect children from 17 different classes was based on the necessity to control for the socio-mathematical practices children experienced previously in their classroom (Verschaffel et al. 2009).

The first four days (Monday to Thursday) the children participated in 90 min treatment sessions in the morning and in the afternoon. The fifth day (Friday) was reserved for the post-test and other activities. Detailed teaching scripts (including learning materials and exercises) were developed based on the theoretical assumptions for the explicit and implicit conditions. Four experienced researchers for arithmetic education from different universities reviewed and approved the content validity of the teaching scripts and the material as well as the appropriateness of the planned interventions for the study goal. Finally, the feasibility of the two interventions was verified in a pilot study.

The characteristic feature of the explicit condition was an explicit introduction of a set of computation strategies by the teacher and a comparison of the strategies' efficiency for given arithmetic problems. In the first three days, the five strategy types presented in Table 1 were successively demonstrated to the children. In each case, the teacher presented and explained the specific strategy and the children practiced this strategy. After the introduction of the second strategy, the teacher additionally implemented group work sessions in which the children were asked to solve given problems efficiently and to compare their solutions. After each of these group work phases different solutions were presented to the entire class. Here, the teacher paid attention that different strategies were reflected by the children concerning their efficiency. Hence, the explicit condition followed the assumptions that for the formation of children's skills in adaptive use of strategies they should develop a strategy repertoire, acquire routine expertise in strategy execution and become aware of a strategy's efficiency for given tasks in the sense of strategy distribution (cf. Chen and Siegler 2000; see also Symons et al. 1989). Following the empirical findings presented above, a comparison of different strategies was implemented as early as possible (Blöte et al. 2001; Durkin et al. 2017; Klein et al. 1998; Rittle-Johnson et al. 2012; RittleJohnson and Star 2007).

The implicit condition was based on the previously elaborated assumptions that an explicit demonstration of strategies is not necessary (e.g., Siegler 2003) or even has negative effects in terms of creativity (Threlfall 2002). Hence, the implicit learning environment followed the suggestion of providing suitable problems (Siegler 2003). Some of the implemented problems addressed conceptual knowledge on numbers (Baroody and Ginsburg 1986; Threlfall 2002) whereas others asked children to invent own efficient strategies 
and gave the opportunity to generalize from a specific problem so that they could associate strategies with problem types (Gravemeijer 2004, p. 118) which is beneficial for the generation effect. In the lessons the children were repeatedly encouraged to solve given problems, to compare their solutions and to discuss the efficiency with respect to problem characteristics (here we used the same addition and subtraction problems as in the explicit condition). Moreover, the children were asked to analyze numerical characteristics of problems (e.g., the relation of the involved numbers) and to categorize problems (without solving them) into apparently "easy problems" (e.g., 250+150), "smart problems" which can be transformed to an easier problem (e.g., 364+299) and "other problems" for which no apparent easy solution exists (e.g., 237+386). In all lessons, the teacher strictly avoided (a) presenting an "official" answer to the questions of efficient solutions for a given problem or problem categorization, and (b) introducing official names for strategies (like stepwise or jump strategy). Hence, the teacher acted as a mentor and provided structured learning opportunities to support children for their individual meaningful generation of procedures and concepts (Baroody 2003, p. 22). The implementation of a socio-mathematical practice which implicitly set norms about preferable strategies by emphasizing advantages of certain strategies or by giving the impression that some strategies are more important than others was avoided (Verschaffel et al. 2009). Instead the teacher reminded children of numerical characteristics of the problems which they can use as criteria to compare the efficiency of their strategies. Moreover, the teacher ensured the invented and applied strategies were mathematically correct (independent of the efficiency).

Table 2 gives a short overview of the activities during the 5 days. As mentioned before, the children came from 17 different classes so that most of them did not know each other and they experienced different mathematics classes before the holiday course. Hence, both conditions started with a review of the decimal system with numbers up to 1000 and the introduction of the method of small group discussions in which children compare their solutions.

\section{Measures}

Strategy types, adaptive and accurate use of strategies Data on the type of children's strategies and on their adaptive and accurate application was collected using four written tests (T1: pretest, T2: posttest, and two follow-up tests T3, T4). Each test consisted of eight multi-digit addition and subtraction tasks suggesting specific strategies as efficient solutions (e.g., compensation, simplifying or indirect addition, Table 1). The children were explicitly asked to solve the problem in a clever way and to write down their calculation. The four tests were similar as we planned to conduct repeated measures analyses: consecutive tests had six common items and four items were included in all tests (403-396, $1000-991,398+441,502+399)$.

Children's solutions were coded by a fine-grained category system with 21 strategy categories (10 of these are listed below in Table 4) so that each solution could be assigned to one strategy category. The categories were developed on the basis of a literature review and from the data in a bottom-up procedure. Children's solutions were coded independently by two trained research assistants who reached an acceptable inter-rater reliability (all Cohen's $\kappa>.70$ ). In case of different coding a consensual agreement was achieved after a discussion.

Each item solution was scored twice: firstly for accuracy as correct (1 point) or incorrect (0 points), and secondly for adaptivity based on the efficiency of the strategy used for the given test item. For the latter, we used the 21 strategy categories: for each test item it was 


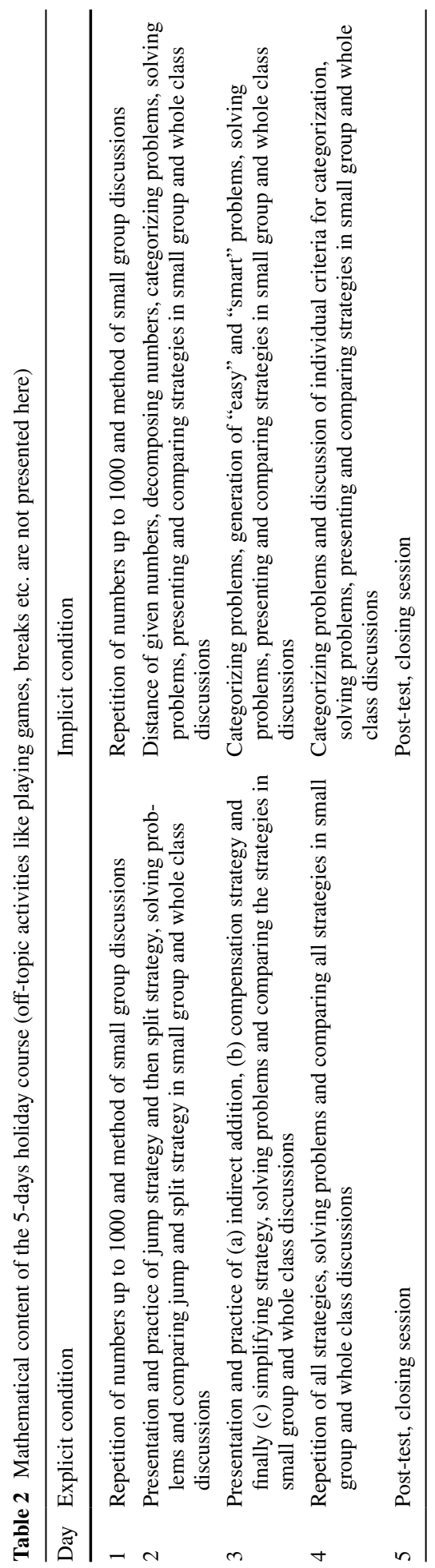


decided which of the 21 categories are classified as adaptive strategies ( 2 points), "partly adaptive" strategies (1 point) or not adaptive strategies (0 points). As already mentioned in the beginning we followed a normative approach for deciding whether a strategy for a given problem is efficient (i.e., chosen adaptively) or not. This decision was guided by two criteria: (a) the mathematical perspective, that is, which strategies provide short solutions and (b) the psychological perspective, that is, which strategies require little mental effort. The latter depends on the knowledge and skills the target population (in our case thirdgraders) has acquired so far. For our specific topic of addition and subtraction with multidigit numbers in grade 3 these criteria allowed a consensual scoring of strategy categories for each test item.

For each measurement point T1-T4 we generated a scale for adaptive use of strategies and one for accurate use of strategies. Due to the large number of common items in the four tests we consider the scales as repeated measures for adaptive and accurate use of strategies respectively. The reliability (Cronbach's $\alpha$ ) of the four scales on adaptive use of strategies (T1-T4) was acceptable or $\operatorname{good}(\alpha=.74, .81, .88$ and .89$)$ whereas the reliability of the four scales on accurate use of strategies (T1-T4) turned out to be between satisfying and $\operatorname{good}(\alpha=.78, .81, .74$ and .66$)$.

Covariate Data for general cognitive abilities was included in the analysis as covariate. General cognitive abilities were assessed in our intervention group sample at $\mathrm{T} 1$ by the classification and the matrix subtest of the German version of the culture fair intelligence test (CFT-1 R, Cattell et al. 1997). IQ scores were computed based on the manual and corresponding norm tables for third-graders.

\section{Data analyses}

\section{Handling of missing data}

The sample was tested four times within 9months (T1-T4 for adaptive and accurate use of strategies, and general cognitive abilities at T1 as covariate). Due to child absences (illness) only 61 of the 73 data sets were complete: one child missed two tests and 11 children one of the five tests. The missing rate from data of adaptive and accurate use of strategies (T1-T4) was $3.77 \%$ (11 missings), whereas the missing rate from the covariate was $2.74 \%$ (two missings). Using only the sample of the 61 children with complete data set would have reduced the experimental groups to 29 children in the explicit condition and 32 in the implicit condition with a substantial loss of information. Hence, when appropriate we applied statistical procedures (linear mixed models) which can handle missing data for the dependent variables. For the two missing values in the covariate (general cognitive abilities) we applied the single imputation procedure in SPSS 23.0.

\section{Statistical data analyses}

Sample selection bias was examined with a $t$ test at T1 for adaptive and for accurate use of strategies (intervention group vs. their classmates).

As intervention check we compared the skill development in adaptive and in accurate use of strategies of our intervention group at T1 and T3 with the corresponding skill development of their classmates. We conducted repeated measures ANCOVAs for T1 and T3 so that only the 67 children of the intervention groups could be included which participated at $\mathrm{T} 1$ and at T3 (one child was absent at T1 and five others at T3). 
We compared the effects of the implicit and the explicit learning environment concerning skills in adaptive and accurate use of strategies (Hypotheses 1 and 2) by applying linear mixed models with post hoc comparisons (Bonferroni correction). Linear mixed models can handle missing data for the dependent variable and avoid listwise deletion in cases of more than two points of measurement. Subjects were treated as random effects to take into account within-subject correlations in the dependent variable. For Hypothesis 1, children's skills in adaptive use of strategies were taken as dependent variable with time (i.e., measurement points T1-T4) as repeated measure. Analogously, we chose children's skills in accurate use of strategies as dependent variable to examine Hypothesis 2. The variables "intervention group" (implicit vs. explicit learning environment), "time", the interaction term "intervention group*time", and the covariate "general cognitive abilities" were included as fixed effects. An autoregressive covariance structure (AR1) for repeated measures was selected for both linear mixed models because it provided the best model fit according to Akaines Information Index (AIC) in case of adaptive as well as in case of accurate use of strategies.

Finally, we compared the effects of the implicit and the explicit learning environment regarding the applied strategies (Hypotheses 3 and 4). Here we used the frequencies in the 21 strategy categories from our coding scheme (see Sect. "Measures"). We conducted $\chi^{2}$-homogeneity tests to compare the two groups of students at each measurement point T1-T4 and used Cramer's $V$ as effect size for the differences. We had to merge strategy categories of the same strategy type to avoid too many small cell frequencies due to prerequisites for the $\chi^{2}$-homogeneity tests so that we finally end up with 10 strategy categories (Table 4). Since we wanted to compare the applied strategies of children from the two learning environments, we ignored cases when a child did not solve an item. This resulted in a different number of strategies for T1-T4 because some children did not process one or more items or children did not participate in one of the tests.

\section{Results}

\section{Sample selection and intervention check}

To control for selection bias we compared the skills of children from the intervention (implicit and explicit learning environment) with those of their classmates which did not participate in the intervention. For skills in adaptive and in accurate use of strategies the children from the intervention showed slightly higher mean values at pretest T1. However, the means did not differ significantly and the effect sizes (Cohen's $d$ ) did not indicate substantial differences so that a selection bias could be excluded (skills in adaptive use of strategies: $M=4.38, S D=3.00$ vs. $M=3.89, S D=3.15$ with $t(249)=1.12, p=.263$, $d=0.16$; skills in accurate use of strategies: $M=4.93, S D=2.37$ vs. $M=4.45, S D=2.04$ with $t(249)=1.62, p=.106, d=0.22)$.

For the intervention check we compared the children of the intervention with their classmates (without intervention) concerning their skill development in adaptive use of strategies from pre-test (T1) to the first follow-up test (T3). ${ }^{1}$ The repeated measures ANOVA

\footnotetext{
1 As mentioned in Sect. "Statistical data analyses" there is no post-test data for the group of classmates because they were on fall break during that time.
} 
yielded a significant interaction effect "time* group" with a moderate effect size in favour of the intervention group $\left(F(1,244)=19.10, p<.001, \eta^{2}=.073\right)$. Hence, the intervention had a positive effect on students' skills in adaptive use of strategies.

\section{Effects of the implicit and explicit learning environment on children's skills in adaptive and accurate use of strategies}

Table 3 presents descriptive results of children skills in the implicit and explicit learning environment at pretest, posttest and the two follow-up tests after 3 and 8 months.

The result of the linear mixed model indicated that there is no significant effect of the type of learning environment (represented by the variable "group") on children's skills in adaptive use of strategies. The interaction effect "time* group" $[F(3,198.136)=0.70$, $p=.550]$ as well as the main effect "group" $[F(1,82.826)=0.40, p=.528]$ did not reach level of statistical significance. Based on the estimated marginal means for children's skills in adaptive use of strategies, we computed Cohen's $d$ as effect sizes for group differences (implicit environment-explicit environment) at T1-T4. The values did not indicate noteworthy effect sizes at T1 and T2 and only small effect sizes at T3 and T4 $\left(d_{T 1}=0.05\right.$, $\left.d_{T 2}=-0.06, d_{T 3}=0.25, d_{T 4}=0.20\right)$.

The linear mixed model for children's skills in accurate use of strategies yielded similar results. Neither the interaction effect "time* group" $[F(3,195.100)=0.23, p=.878]$ nor the main effect "group" $[F(1,81.603)=0.73, p=.395]$ reached a level of statistical significance. Here, we got effect sizes for group differences (implicit environment-explicit environment) which can be classified as small at T1 and T2 and not noteworthy at T3 and T4 $\left(d_{T 1}=0.24, d_{T 2}=0.22, d_{T 3}=0.12, d_{T 4}=0.01\right)$.

Hence, Hypothesis 1, that children in the implicit learning environment show an overall better adaptive use of strategies than the children in the explicit learning environment-as a short- and long-term effect-had to be rejected. In contrast, the Hypothesis 2 that both groups do not differ in the accurate use of strategies could be confirmed.

\section{Effects of the learning environments on strategy types children use}

In Table 4 we present the distribution of all strategies the children from the implicit and the explicit learning environment used in the tests at T1-T4. We compared the applied strategies for each test by running a $\chi^{2}$-homogeneity test. It turned out that the two groups did not differ significantly before the intervention $\left[\mathrm{T} 1: \chi^{2}(8, N=512)=5.39, p=.716\right.$, Cramér's $V=.10]$ whereas there are significant differences with medium effect sizes at posttest $\left[\mathrm{T} 2: \chi^{2}(9, N=552)=94.82, p<.001, V=.41\right]$ as well as at the follow-up tests [T3: $\left.\chi^{2}(9, N=529)=69.13, p<.001, V=.36 ; \mathrm{T} 4: \chi^{2}(9, N=533)=56.94, p<.001, V=.33\right]$. Focussing the specific strategies of the types indirect subtraction and simplifying it turned out that after the intervention at posttest (T2) both groups used these advanced strategy types with an increased frequency. As displayed in Table 4, children from the explicit learning environment used these strategies more than twice as often as children from the implicit learning environment (indirect subtraction type: 61 vs. 26; simplifying type: 45 vs. 18). A similar distribution can be seen regarding the number of children using these strategies (indirect subtraction type: 22 children vs. 10 children; simplifying type: 16 vs. 12, not displayed in Table 4), although the difference between the two groups is slightly smaller. Overall, these results support Hypothesis 3. 


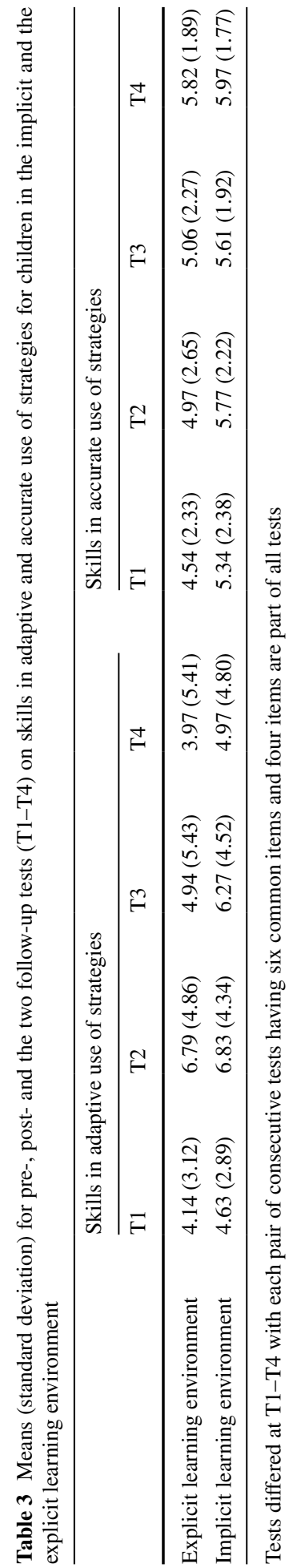




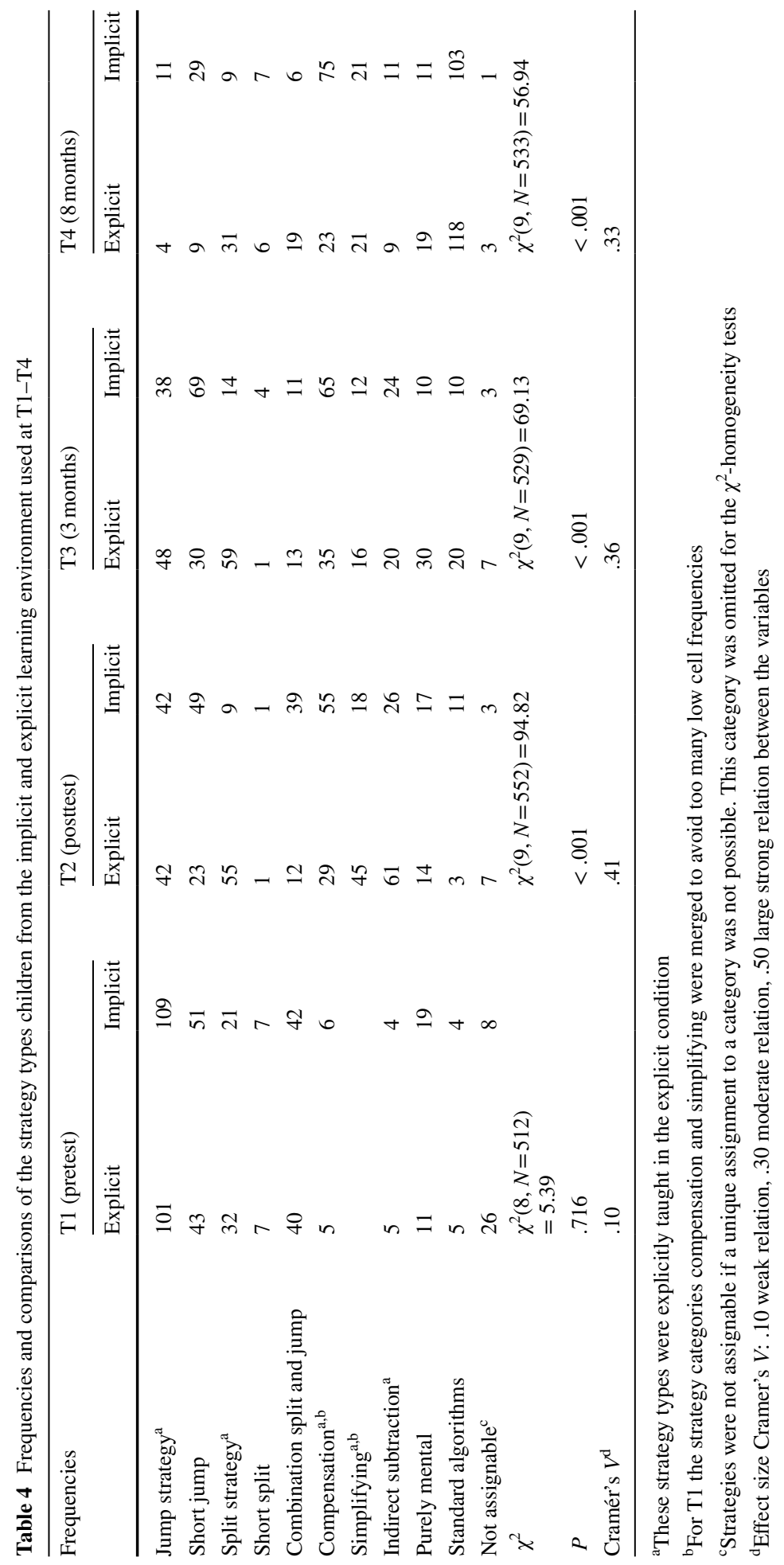


Conversely, the children from the implicit learning environment used more frequently strategies of the compensation type at posttest (55 vs. 29) which is also mirrored by the number of children using a compensation type strategy (15 vs. 10) at least once. This advance in using the compensation type strategies for the children from the implicit learning environment is sustainable at the follow-up tests after 3 months (T3, frequency: 65 vs. 35, children: 13 vs. 10) and after 8 months (T4, frequency: 75 vs. 23 , children: 16 vs. 5). The frequency of this strategy type even increased from T2-T4 and "resisted" the introduction of the standard algorithms between T3 and T4. Thus, the findings support Hypothesis 4 for the compensation type strategies but not for the other strategy types.

\section{Discussion}

Summarizing the results, we firstly did not find significant differences between the effects of the implicit and explicit learning environment for children's skills in adaptive and accurate use of strategies. We expected this result for children's skills in accurate use of strategies (Hypothesis 2). Unlike we hypothesized, children from both groups did also not differ significantly in their performance in adaptive use of strategies, i.e., in providing efficient strategies for multi-digit addition and subtraction problems. Despite the rejection of Hypothesis 1, we secondly identified different effects of the implicit and the explicit learning environment on children's use of strategies. As expected by Hypothesis 3, children taught by the explicit learning environment more frequently used advanced strategies of the types simplifying and indirect subtraction in the posttest than their counterparts from the implicit learning environment. In contrast, children taught by the implicit learning environment used newly learned strategies (like the compensation type) more sustainable than their counterparts from the explicit learning environment which partly supports Hypothesis 4.

\section{Theoretical implications}

Our study supplements research on children's development of skills in adaptive use of strategies, particularly for the phase of strategy acquisition. As elaborated in the theoretical background, several authors (e.g., Baroody 2003; Gravemeijer 2004; Siegler 2003; Threlfall 2002, 2009) assume that (1) children can acquire new strategies by invention as long as they are confronted with suitable problems, and (2) such a learning environment allows children to increase strategy fluency and to reduce cognitive load so that children can improve their skills in adaptive use of strategies by metacognitive processes. Overall our findings support these assumptions. Children taught in the implicit learning environment were repeatedly confronted with suitable problems in order to generate new strategies and to increase fluency of their strategies. Moreover, they were asked to examine the efficiency of their strategies by strategy comparison which gave opportunities for metacognitive processes. It turned out that these children improved their skills in adaptive use of strategies during the intervention and that their skill development did not differ compared to children who participated in lessons based on a systematic and explicit teaching of adaptive use of strategies. These results indicate that-even for three-digit addition and subtraction problems - children are able to self-invent different strategies and to learn their adaptive use. However, the findings do not confirm the generation effect for children's skill development 
in this complex learning content because learning by generation in the implicit condition was not more effective than learning by presentation in the explicit condition.

In addition, our investigation of the types of strategies children used in the posttest directly after the intervention uncovered a noteworthy difference between the children of the implicit and the explicit learning environment. Though both groups of children find efficient solutions to the problems they differed in the used strategy types (cf. Table 4). Despite the presentation of suitable problems as learning opportunities, for most children in the implicit learning environment it was too challenging to generate some of the taskspecific and highly efficient strategies, to adopt these to the individual strategy repertoire, and to develop an association of the strategy to certain problem types. Thus, the necessary condition for the generation effect, that is, the generation of strategies by the students, was not satisfied for these challenging task-specific strategies. In line with the results of Chen et al. $(2015,2016)$, our findings suggest that for complex content the demonstration by the teacher or material is more effective than the generation by students. The reverse is true for easier task-specific strategies (the compensation type strategies in our study), which is consistent with the results of Rittle-Johnson and Kmicikewycz (2008): Here, our findings support the generation effect because children from the implicit learning environment used the compensation type strategies in the post- and the follow-up tests with increasing frequency and more frequently than children from the explicit learning environment (Table 4). Especially, the observed effect in the delayed tests is in line with other research studies on the generation effect (cf. Chen et al. 2016). Overall, for research in instructional science addressing strategy acquisition and the adaptive use of strategies, our results specifically suggest to take into account whether students are able to generate the target strategies or not. It seems that this is one of the crucial factors for the question whether the generation effect comes into play and whether an environment based on learning by generation is effective or not.

In addition to the implications on strategy learning in general, our results specifically provide insight for research on children's acquisition of multi-digit addition and subtraction strategies. For the special case of the indirect addition strategy (which belongs to the strategy type indirect subtraction, cf. Table 1), our study replicates the results of De Smedt et al. (2010) and Torbeyns et al. (2009c). These findings seem to question the idea that the indirect addition strategy can be self-invented by third graders based on suitable problems. Other studies show that young adults are able to use the indirect addition adaptively (e.g., Peters et al. 2010, 2012; Torbeyns et al. 2009e), although we can assume that this strategy was hardly addressed explicitly in their mathematics classroom. Overall, it seems that for the acquisition of advanced strategies, like strategies of the indirect subtraction type or the simplifying type either a continuous and explicit learning environment is necessary or-in case of an implicit learning environment-students need specific individual prerequisites before they can self-invent these strategies. Both assumptions are plausible in the context of an explanation suggested by Schneider and Stern (2009). They argue that children's knowledge is fragmented as they often fail to see abstract relations in arithmetic. If we consider the two advanced strategy types simplifying and indirect subtraction, then it becomes clear that the underlying mathematical relations are challenging. In the case of indirect addition we refer to several studies and analyses which examined the complexity of this specific strategy especially addressing the inverse principle and complement principle (e.g., the special issue Verschaffel and Torbeyns 2009). The strategy type simplifying is a similar challenge for young learners: a child must accept the equivalence of two expressions $(527+398=525+400$ or $527-398=529-400)$, that is it must accept that the result of the two problems is identical without knowing this result. Though this strategy 
type looks similar to the type compensation strategy (cf. Table 1), the idea of both strategy types is different. In case of the compensation strategy only one of the addends will be changed and this change is compensated (e.g., $527+398=527+(400-2)=527+400$ - 2). Consequently, the underlying abstract principle is much easier than for the simplifying strategy. As our findings show, many children in the implicit learning environment are able to invent and to adaptively use the compensation strategy. Hence, the explanation of Schneider and Stern (2009) fits to our empirical results. Children from the explicit learning environment were better able to adopt especially advanced strategies to their strategy repertoire and associate these strategies to problem types (as the posttest results show). Since they were not able to understand the underlying abstract principles of the advanced strategies, the corresponding fragments of strategy knowledge were not stable (as the follow-up results show). Children from the implicit learning environment were less successful in the acquisition of advanced strategies. An invention of these strategies is too challenging for most of the children because they need to understand the underlying abstract mathematical principles. Accordingly, in the implicit learning environment alternative strategies based on easier mathematical principles were generated (e.g., compensation type strategies) and adopted to children's strategy repertoire. Interestingly, the generation effect was quite sustainable in this case because 8 months after the intervention and after the introduction of the standard algorithms for addition and subtraction in the regular mathematics classroom, almost half of the children (16 from 35) taught by the implicit learning environment group still used the compensation strategy in the second follow-up test. This result is surprising because we can assume that in the socio-mathematical context of the mathematics classroom (Verschaffel et al. 2009) especially the standard algorithms belong to the highly valued strategies for multi-digit addition and subtraction problems. It seems that the invention and practicing of specific strategies provide the opportunity for a strong association between strategies and problem types (in the sense of Shrager and Siegler 1998). Hence, this avoids a quick replacement of these strategies by new strategies (i.e., standard algorithms) which are less efficient for the considered problem types but highly valued in the socio-mathematical context of the current mathematics classroom.

\section{Educational implications}

Our study provides different kinds of educational implications. First of all, our results indicate that teaching adaptive use of strategies is not accompanied by a decrease of children's strategy accuracy. Especially for arithmetic we cannot confirm the fear of teachers that learning environments which foster the flexible use of different strategies will overwhelm students with too much information (e.g., Silver et al. 2005).

Second, as elaborated upon in the previous section, it seems that teaching adaptive use of strategies needs to provide continuous learning opportunities for advanced strategies (like indirect addition or simplifying in our study). Based on the ideas of Baroody (2003) as well as Schneider and Stern (2009), it could be promising to follow a knowledge integration perspective and to establish teaching methods providing both conceptual and procedural knowledge. Our findings are in line with other results showing that it is quite challenging for children in primary school grades to self-invent advanced strategies. Accordingly, an explicit teaching of these strategies might be a fruitful possibility. However, to avoid non-sustainable effects like in our intervention group taught by the explicit learning environment, it is necessary to provide additional learning opportunities for knowledge integration. Especially, the understanding of underlying principles seems to be 
necessary to integrate knowledge fragments and to provide a stronger association between strategy and problem type. As an alternative approach, it might also be possible to develop specific implicit learning environments effective for advanced strategies. For example, in a study of van den Heuvel-Panhuizen (1996) addressing arithmetic strategies, children used the indirect addition strategy when they got contextualized (word) problems instead of bare number problems (like in our study). In particular, the everyday context might be advantageous to induce the invention of advanced strategies. However, it is not clear if children also will apply these strategies for decontextualized problems (e.g., for bare number problems in arithmetic, cf. the discussion in De Smedt et al. 2010).

\section{Limitations of the study}

For the interpretation of the results of our study, some limitations should be kept in mind. First, our study was organized as a controlled experiment and included a small sample with $N=73$ third-graders which reduced the power for detecting differences between groups. Accordingly, for our analysis of group differences we also considered effect sizes in addition to level of significance though we are aware that this information cannot fully compensate the small sample size. Second, we tried to control the pre-knowledge and experience of the third-graders in our sample with respect to adaptive use of strategies. Nevertheless, we did not know what happened in the mathematics classroom in second grade. Similarly, we had no influence over the mathematics class after our intervention during fall break. This means, that we did not know how the children learned arithmetic computations between posttest (T2) and the follow-up tests (T3, T4). Since the children of our sample were distributed over 17 classes and showed much better skills in adaptive use of strategies than their classmates after 3 months at T3 (see Sect. "Sample selection and intervention check"), we think that the regular mathematics class in the 17 classes did not intensively foster adaptive use of strategies. Third, like other studies we assessed children's strategy use using written tests and asked the children to find a clever solution and write down their "calculation". Each of these tests was restricted to only eight items which ensured an acceptable test motivation of the third graders but restricted the available data. In the data analysis we assumed that the written solutions reflected the strategy the children applied for solving the items but we cannot be sure that this was really the case. Finally, we can state that our teaching sessions in the intervention were organized in a similar manner to the ordinary mathematics teaching in primary schools. However, the compact character of our intervention (16 lessons in 1 week instead of 4 weeks) clearly reduced the ecological validity. Hence, a possible transfer of the results to educational practice should be considered with caution.

Funding The research presented in the manuscript was funded by Grants "HE 4561/3-3" and "LI 1639/13" from the Deutsche Forschungsgemeinschaft (German Research Foundation, DFG).

Open Access This article is distributed under the terms of the Creative Commons Attribution 4.0 International License (http://creativecommons.org/licenses/by/4.0/), which permits unrestricted use, distribution, and reproduction in any medium, provided you give appropriate credit to the original author(s) and the source, provide a link to the Creative Commons license, and indicate if changes were made. 


\section{References}

Baroody, A. J. (2003). The development of adaptive expertise and flexibility: The integration of conceptual and procedural knowledge. In A. J. Baroody \& A. Dowker (Eds.), The development of arithmetic concepts and skills (pp. 1-34). Mahwah: Erlbaum.

Baroody, A. J., \& Ginsburg, H. P. (1986). The relationship between initial meaningful and mechanical knowledge of arithmetic. In J. Hiebert (Ed.), Conceptual and procedural knowledge: The case of mathematics (pp. 75-112). Hillsdale: Erlbaum Associates.

Baroody, A. J., \& Tiilikainen, S. H. (2003). Two perspectives on addition development. In A. J. Baroody \& A. Dowker (Eds.), The development of arithmetic concepts and skills: Constructing adaptive expertise (pp. 75-125). Mahwah: Lawrence Erlbaum Associates.

Beishuizen, M. (1993). Mental strategies and materials or models for addition and subtraction up to 100 in Dutch second grades. Journal for Research in Mathematics Education, 24(4), 294-323.

Bertsch, S., Pesta, B. J., Wiscott, R., \& McDaniel, M. A. (2007). The generation effect: A meta-analytic review. Memory and Cognition, 35(2), 201-210.

Blöte, A. W., Klein, A. S., \& Beishuizen, M. (2000). Mental computation and conceptual understanding. Learning and Instruction, 10, 221-247.

Blöte, A. W., Van der Burg, E., \& Klein, A. S. (2001). Students' flexibility in solving two-digit addition and subtraction problems: Instruction effects. Journal of Educational Psychology, 93, 627-638.

Carpenter, T. P., Franke, M. L., Jacobs, V. R., Fennema, E., \& Empson, S. B. (1997). A longitudinal study of invention and understanding in children's multidigit addition and subtraction. Journal for Research in Mathematics Education, 29(1), 3-20.

Cattell, R., Weiß, R., \& Osterland, J. (1997). Grundintelligenztest Skala 1. CFT 1. Göttingen: Hogrefe.

Chen, O., Kalyuga, S., \& Sweller, J. (2015). The worked example effect, the generation effect, and element interactivity. Journal of Educational Psychology, 107(3), 689-704.

Chen, O., Kalyuga, S., \& Sweller, J. (2016). Relations between the worked example and generation effects on immediate and delayed tests. Learning and Instruction, 45, 20-30.

Chen, Z., \& Siegler, R. S. (2000). Across the Great Divide: Bridging the Gap Between Understanding of Toddlers' and Older Children's Thinking. In Monographs of the Society for Research in Child Development, 65 (2, Serial No. 261).

Crowley, K., \& Siegler, R. S. (1993). Flexible strategy use in young children's tic-tac-toe. Cognitive Science, 17, 531-561.

Csíkos, C. (2016). Strategies and performance in elementary students' three-digit mental addition. Educational Studies in Mathematics, 91(1), 123-139.

De Smedt, B., Torbeyns, J., Strassens, N., Ghesquière, P., \& Verschaffel, L. (2010). Frequency, efficiency and flexibility of indirect addition in two learning environments. Learning and Instruction, 20, 205-215.

Durkin, K., Star, J. R., \& Rittle-Johnson, B. (2017). Using comparison of multiple strategies in the mathematics classroom: lessons learned and next steps. ZDM Mathematics Education, 49, 585-597.

Gardner, W. P., \& Rogoff, B. (1990). Children's deliberateness of planning according to task circumstances. Developmental Psychology, 26, 480-487.

Gravemeijer, K. (2004). Local instruction theories as means of support for teachers in reform mathematics education. Mathematical Thinking and Learning, 6(2), 105-128.

Heinze, A., Marschick, F., \& Lipowsky, F. (2009). Addition and subtraction of three-digit numbers: Adaptive strategy use and the influence of instruction in German third Grade. ZDM: International Journal on Mathematics Education, 41(5), 591-604.

Klayman, J. (1985). Children's decision strategies and their adaptation to task characteristics. Organizational Behavior and Human Decision Processes, 35(2), 179-201.

Klein, A. S., Beishuizen, M., \& Treffers, A. (1998). The empty number line in Dutch second grades: Realistic versus gradual program design. Journal for Research in Mathematics Education, 29, 443-464.

Lemaire, P., \& Siegler, R. S. (1995). Four aspects of strategic change: Contributions to children's learning of multiplication. Journal of Experimental Psychology: General, 124, 83-97.

McNamara, D. S., \& Healy, A. F. (2000). A procedural explanation of the generation effect for simple and difficult multiplication problems and answers. Journal of Memory and Language, 43, 652-679.

Peters, G., De Smedt, B., Torbeyns, J., Ghesquière, P., \& Verschaffel, L. (2010). Adults' use of subtraction by addition. Acta Psychologica, 135, 323-329.

Peters, G., De Smedt, B., Torbeyns, J., Ghesquière, P., \& Verschaffel, L. (2012). Children's use of subtraction by addition on large single digit subtractions. Educational Studies in Mathematics, 79, 335-349. 
Rittle-Johnson, B., \& Kmicikewycz, A. O. (2008). When generating answers benefits arithmetic skill: The importance of prior knowledge. Journal of Experimental Child Psychology, 101(1), 75-81.

Rittle-Johnson, B., \& Star, J. R. (2007). Does comparing solution methods facilitate conceptual and procedural knowledge? An experimental study on learning to solve equations. Journal of Educational Psychology, 99, 561-574.

Rittle-Johnson, B., Star, J. R., \& Durkin, K. (2012). Developing procedural flexibility: When should multiple solution procedures be introduced? British Journal of Educational Psychology, 82, 436-455.

Schneider, M., \& Stern, E. (2009). The inverse relation of addition and subtraction: A knowledge integration perspective. Mathematical Thinking and Learning, 11(1-2), 92-101.

Selter, C. (2001). Addition and subtraction of three-digit numbers: German elementary children's success, methods, and strategies. Educational Studies in Mathematics, 47, 145-173.

Shrager, J., \& Siegler, R. S. (1998). SCADS: A model of children's strategy choices and strategy discoveries. Psychological Science, 9, 405-410.

Siegler, R. S. (1996). Emerging minds. New York: Oxford University Press.

Siegler, R. S. (2003). Implications of cognitive science research for mathematics education. In J. Kilpatrick, W. B. Martin, \& D. E. Schifter (Eds.), A research companion to principles and standards for school mathematics (pp. 219-233). Reston: National Council of Teachers of Mathematics.

Silver, E. A., Ghousseini, H., Gosen, D., Charalambous, C., \& Strawhun, B. (2005). Moving from rhetoric to praxis: Issues faced by teachers in having students consider multiple solutions for problems in the mathematics classroom. Journal of Mathematical Behavior, 24, 287-301.

Slamecka, N. J., \& Graf, P. (1978). The generation effect: Delineation of a phenomenon. Journal of Experimental Psychology: Human Learning and Memory, 4, 592-604.

Symons, S., Snyder, B. L., Cariglia-Bull, T., \& Pressley, M. (1989). Why be optimistic about cognitive strategy instruction? In C. B. McCormick, G. E. Miller, \& M. Pressley (Eds.), Cognitive strategy research (pp. 3-32). New York: Springer.

Threlfall, J. (2002). Flexible mental calculation. Educational Studies in Mathematics, 50(1), $29-47$.

Threlfall, J. (2009). Strategies and flexibility in mental calculation. ZDM: The International Journal on Mathematics Education, 41(5), 541-555.

Torbeyns, J., De Smedt, B., Ghesquière, P., \& Verschaffel, L. (2009a). Acquisition and use of shortcut strategies by traditionally schooled children. Educational Studies in Mathematics, 71(1), 1-17.

Torbeyns, J., De Smedt, B., Ghesquière, P., \& Verschaffel, L. (2009b). Jump or compensate? Strategy flexibility in the number domain up to 100. ZDM: The International Journal on Mathematics Education, 41(5), 581-590.

Torbeyns, J., De Smedt, B., Ghesquière, P., \& Verschaffel, L. (2009c). Solving subtractions adaptively by means of indirect addition: Influence of task, subject, and instructional factors. Mediterranean Journal for Research in Mathematics Education, 8, 1-30.

Torbeyns, J., De Smedt, B., Stassens, N., Ghesquière, P., \& Verschaffel, L. (2009d). Solving subtraction problems by means of indirect addition. Mathematical Thinking and Learning, 11(1-2), 79-91.

Torbeyns, J., Ghesquière, P., \& Verschaffel, L. (2009e). Efficiency and flexibility of indirect addition in the domain of multi-digit subtraction. Learning and Instruction, 19(1), 1-12.

Torbeyns, J., \& Verschaffel, L. (2016). Mental computation or standard algorithm? Children's strategy choices on multi-digit subtractions. European Journal of Psychology of Education, 31(2), 99-116.

Torbeyns, J., Verschaffel, L., \& Ghesquière, P. (2006). The development of children's adaptive expertise in the number domain 20-100. Cognition and Instruction, 24, 439-465.

Van den Heuvel-Panhuizen, M. (1996). Assessment and realistic mathematics education. Utrecht: CD- $\beta$ Press/Freudenthal Institute, Utrecht University.

Verschaffel, L., Luwel, K., Torbeyns, J., \& Van Dooren, W. (2009). Conceptualizing, investigating, and enhancing adaptive expertise in elementary mathematics education. European Journal of Psychology of Education, 24, 335-359.

Verschaffel, L. \& Torbeyns, J. (Eds.) (2009). Young children's understanding and application of subtractionrelated principles. Mathematical Thinking and Learning, 11. Special issue. 